\title{
Propriedade microestrutural do refinamento da liga amorfa $\mathrm{Al}_{54} \mathrm{Nb}_{45} \mathrm{~B}_{5}$ por moagem mecânica
}

\section{Microstructural property refinement amorphous alloy $\mathrm{Al}_{54} \mathrm{Nb}_{45} \mathrm{~B}_{5}$ by mechanical milling}

\author{
Luciano Nascimento ${ }^{1}$
}

\begin{abstract}
Resumo
A presença de Nióbio na liga cria regiões de fases intermetálicas contido na liga $\mathrm{Al}_{54} \mathrm{Nb}_{45} \mathrm{~B}_{5}$ amorfa que torna um potente substrato na nucleação heterogênea para a nucleação de dendríticos com a presença de óxidos de Alumínio devidos aos processos difusivos em superfície da liga amorfa $\mathrm{Al}_{54} \mathrm{Nb}_{45} \mathrm{~B}_{5}$. Esta liga é extremamente de custo relativamente baixo, porém com aplicações em muitos setores da indústria química e petroquímica em ênfase, pois a sua deformação mecânica é alta devido à presença de $(\mathrm{Nb}$ ou $\mathrm{B})$ no alumínio atuando, principalmente, como refinadores de grão para melhorar suas propriedades mecânicas e térmicas usando a moagem de alta energia. O emprego combinado de Nióbio e Boro (fases intermetálicas são formadas por meio de adição de pós de $\mathrm{Al}$ e $\mathrm{Nb}$ ), em vez de nióbio ou de boro, individualmente, é uma forma altamente eficaz para refinar o tamanho do grão de liga de Al-Nb-B na sua microestrutura, sem causar inconvenientes na sua rede por deformações indesejadas. Com esse intuito o presente trabalho comparou o efeito do refino do grão promovido pela adição de Nióbio e pela adição do Boro na $\mathrm{Al}_{54} \mathrm{Nb}_{45} \mathrm{~B}_{5}$ obtida por moinho de alta energia sob atmosfera de argônio e caracterizada por Difratometria de Raios X (DRX), Microscopia Eletrônica de Varredura e Energia Dispersiva (MEV/EDS).
\end{abstract}

Palavras-chave: Liga Amorfa. Refinamento Microestrutural. Moagem Mecânica.

\begin{abstract}
The presence of Niobium in the alloy creates regions of intermetallic phases contained in $\mathrm{Al}_{54} \mathrm{Nb}_{45} \mathrm{~B}_{5}$ amorphous alloy which becomes a potent heterogeneous nucleation on the substrate for nucleation with dendritic presence of aluminum oxides due to diffusive processes on the surface of the amorphous alloy $\mathrm{Al}_{54} \mathrm{Nb}_{45} \mathrm{~B}_{5}$. This alloy is extremely low cost and can be applied in many sectors of the chemical and petrochemical industry because its mechanical deformation is high due to the presence of $(\mathrm{Nb}$ or B) in the aluminum acting mainly as grain refiners to improve their mechanical and thermal properties by using the mechanical alloying. The combined use of Niobium and Boron (intermetallic phases are formed by adding $\mathrm{Al}$ and $\mathrm{Nb}$ powder), instead of Niobium or Boron, individually, is a highly effective way to refine the grain size of alloy $\mathrm{Al} \mathrm{Nb-B}$ in its microstructure without causing inconvenience in lattice for unwanted deformations. For this purpose, the present study compares the effect of grain refining promoted by the addition of Niobium and Boron in $\mathrm{Al}_{54} \mathrm{Nb}_{45} \mathrm{~B}_{5}$ obtained by high energy mill under argon atmosphere and characterized by $\mathrm{X}$ rays Diffraction (XRD), Scanning Electron Microscopy and Energy Dispersive (SEM/EDS).
\end{abstract}

Keywords: Amorphous alloy. Microstructural Refinement. Mechanical Milling.

${ }^{1}$ Doutor em Engenharia Química, Programa de Pós-Graduação em Engenharia Química, Centro de Tecnologia e Geociências-CTG/ UFPE. luciano.ufpe@gmail.com 


\section{Introdução}

O alumínio é um metal leve $\left(\rho=2,70 \mathrm{~g} / \mathrm{cm}^{3}\right)$, resistente à corrosão, bom condutor de calor e eletricidade, possui brilho e ponto de fusão de $660,3{ }^{\circ} \mathrm{C}$ e ebulição de $2 \cdot 470^{\circ} \mathrm{C}$, com configuração eletrônica [Ne] $3 \mathrm{~s}^{2} 3 \mathrm{p}^{1}$ pertencente ao grupo 13 (III A), período 3,bloco p e estrutura cristalina de cúbico de face centrada. O alumínio forma compostos somente no estado de oxidação +3 . Seu hidróxido é anfótero, sendo uma substância gelatinosa de cor branca possuidora de um grande poder de adsorção. Soluções contendo o íon $\mathrm{Al}^{3+}$ são incolores, possui baixa resistência à tração $\left(\sigma_{\mathrm{r}}\right)$, cerca de 90 $\mathrm{MPa}$, podendo alcançar $180 \mathrm{Mpa}$. O alumínio foi descoberto por Sir Humphrey Davy em 1809, tendo sido isolado pela primeira vez em 1825 por H.C. Oersted. Sendo o elemento mais abundante, alumínio, na crosta terrestre na forma de óxido de alumínio $\left(\mathrm{Al}_{2} \mathrm{O}_{3}\right)$ e as reservas minerais são quase ilimitadas. O alumínio é o terceiro elemento mais encontrado na crosta terrestre e o mais abundante entre os elementos metálicos. Suas propriedades, como leveza, alta condutividade elétrica, grande resistência à corrosão e baixo ponto de fusão, permitem que seja utilizado de forma extensiva para a produção de diversos itens, tais como ligas metálicas, laminados e extrusão a quente ou a frio.

Ligas amorfas base de alumínio, estão sendo investigadas intensivamente devido às suas propriedades mecânicas, físicas e químicas. Geralmente, ligas amorfas ricas em $\mathrm{Al}$ são submetidas a um processo de cristalização em duas fases mediante aquecimento (INOUE, 1998). A primeira transformação envolve cristalização primária de fases Al-fcc dentro da matriz amorfa. A segunda transformação envolve desvitrificação residual da matriz amorfa em vários compostos intermetálicos. Observou-se que a cristalização preliminar da fase Al-fcc ocorre em resfriamento rápido em ligas de $\mathrm{Al}-\mathrm{Ni}-\mathrm{Ce}(-\mathrm{Cu})$ e em vidros metálicos do tipo Al-Ni-La levando particionamento do soluto (HONO et al., 1995). A diminuição da solubilidade do soluto dos átomos no cristal induz em regiões de soluto enriquecida em torno do cristal. Isto influencia a cinética do crescimento dos cristais de Al-fcc no interior da matriz amorfa. Em algumas ligas amorfas ricas em Al, podem formar fases metaestáveis. A existência de fases metaestáveis em ligas de Al-Ni-RE depende do raio atômico da terra rara. Quanto menor for o raio atômico do elemento de terras raras, maior é a probabilidade da formação destas fases metaestáveis. No sistema de Al-NiLa, uma fase metaestável do tipo bcc dependendo da concentração de Ni e La,isto pode ocorrer (BAREKAR, 2006). Diversos estudos mostram que, dependendo da taxa de resfriamento, podem ser obtidas estruturas mistas em escala manométrica composto por partículas de Al-fcc e por uma matriz amorfa (SAHOO et al., 2005). O tamanho e a variação da fração volumétrica das nanopartículas de Al-fcc também são influenciados pela taxa de resfriamento. Este processo conduz a um aumento na resistência à tração, com tensão máxima de 1560 MPa em aproximadamente $25 \%$ de deformação para um tamanho médio de grão entre 3 e $5 \mathrm{~nm}$. Estas ligas fundidas são materiais comuns usados para fabricar componentes de engenharia para os setores de transporte, especialmente o automotivo, devido à facilidade de sua formação por meio de processos de fundição ea redução intrínseca do peso dos componentes estruturais que envolvem o seu emprego (NOWAK et al., 2015). Além disso, o requisito estrito para a redução do consumo de combustível e, performances mecânicas, portanto, a poluição esgotado gás, bem como a concepção de componentes estruturais com menor peso e avançados estão empurrando a indústria automotiva para o emprego de uma maior quantidade de metais leves, e Al vai definitivamente ter um papel importante (NOWAK; BOLZONI; BABU, 2015). As ligas amorfas formam um grupo de materiais metálicos não cristalinos caracterizados por não possuírem longa distância, uma estrutura atômica ordenada, como na maioria dos cristais (NASCIMENTO, 2013). O princípio básico de obtenção destes materiais é por solidificação rápida de metais a partir de seu estado fundido. Nestes processos, as taxas de resfriamento devem ser elevado, suficiente para que a solidificação ocorra sem haver a cristalização. 
As ligas nanocristalinas, por sua vez, são obtidas a partir das ligas amorfas, e receberam a denominação nanocristalina devido à sua estrutura de grãos da fase $\mathrm{Co}-\mathrm{Nb}-\mathrm{Si}$ em escala nanométrica, na ordem de 1 a $100 \mathrm{~nm}$. É bem conhecido que uma maneira para melhorar as propriedades mecânicas estáticas e dinâmicas de metais é conseguindo estruturas de grão fino (MCCARTNEY, 1980). Os compostos intermetálicos amorfos formados a partir da reação de nióbio $(\mathrm{Nb})$ presente no refinador do grão e do Al da liga Al-Nb-B, formam soluções supersólidas com várias fases que são de interesse em nível termodinãmicos e em processos catalíticos, sendo pouco estudados vários investigadores (BOLZONI; NOWAK; BABU, 2015). Consequentemente, Nb-B inoculação não deve apresentar nenhum efeito de envenenamento. Suas propriedades mecânicas das ligas Al-Si devem ser melhoradas, pois o alongamento é significativamente melhor em ligas Al-Si com adição $\mathrm{Nb}-\mathrm{B}$ formando regiões de fases intermetálicas. Partículas intermetálicas são menores em amostras com adição de $\mathrm{Nb}-\mathrm{B}$, formando regiões amorfizáveis. Macroporosidade reduzido é perceptível em ligas com a adição de $\mathrm{Nb}-\mathrm{B}$ (SIGWORTH, 1984). Esta porosidade reduzida poderia ser reduzida usando o $\mathrm{KBF}_{4}$ adição de sal; no entanto com a utilização de Al-Nb-B liga principal da fração de porosidade é ainda menor, também a porosidade encolhimento é minimizado. O fenômeno de macrosegregação de soluto está relacionado com diferenças na composição química em ordem macroscópica em amostras de Al-Nb-B, o qual pode apresentar diferenças significantes entre regiões internas e externas. Segregação positiva e negativa são desvios na média da composição, onde a segregação positiva significa mais alto conteúdo de soluto na liga e a negativa significa menor concentração, em relação à composição nominal rica em composto intermetálicos de Nb-B e Al-Nb (AL-ZAIN; KIM; MIYAZAKI, 2015). Em condições de crescimento dendrítico, os canais interdendríticos contêm líquido rico em soluto devido à difusão lateral de soluto de suas fases. Associado à contração, o líquido rico em soluto é solidificado em direção à interface livre da base do braço dendrítico, produzindo alta concentração de soluto nas regiões mais externas. Ligas de Al-Nb-B tipicamente apresentam esse comportamento, o qual é conhecido como segregação inversa. As morfologias macro e microestruturais apresentam forte influência na resistência à corrosão e também de microestruturas de ligas de alumínio (JIA et al., 2015; CAI et al., 2015). Geralmente, o processo de resistência à corrosão depende da taxa de resfriamento imposta durante a solidificação, que afeta no tamanho dos braços dendríticos, na redistribuição de soluto, e no comportamento eletroquímico do soluto e solvente dependendo de como são distribuídos. Vale ressaltar que $\mathrm{Nb}$ é um dos elementos com a restrição de maior fator de crescimento, que desempenha um papel importante no refinamento de $\mathrm{Al}$ por meio de nucleação heterogênea (LI et al., 2008). O mecanismo por trás do refinamento de grão de $\mathrm{Al}$ tem sido um tema de debate e foram propostas várias teorias: diagrama de fases / teoria peritética, teoria da hipernucleação e do soluto. Resumindo, o emprego comercia da ligal Al-Nb-B, é com base em pesquisa científica, pois B reage com o $\mathrm{Nb}$ e $\mathrm{Al}$, formando $\mathrm{AlB}_{2}$ e $\mathrm{Nb}_{3} \mathrm{~B}$ ricas em fases intermetálicas. Portanto, o objetivo deste trabalho é apresentar e discutir o desenvolvimento de Al-Nb-B ligas principais com foco na caracterização dos fenômenos que ocorrem durante a sua produção, onde as ligas de Al-Nb-B são usadas para introduzir inoculantes $\mathrm{Nb}-\mathrm{B}$ em diferentes materiais baseados em Al e Si, a fim de avaliar a sua potência de refinamento do grão e de sua microsestrutura. As propriedades mecânicas das ligas de alumínio são fortemente dependentes do efeito macrosegregação dendritítica. Esta liga é extremamente de custo relativamente baixo, porém com aplicações em muitos setores da indústria química e petroquímica. $\mathrm{O}$ intensivo estudo de ligas amorfas à base de alumínio nas últimas décadas continua a busca de composições adequadas e o desenvolvimento de processos para a produção de peças amorfas volumosas, fator esse que abre o caminho para estudos de consolidação de fitas 
ou pós-amorfos em temperaturas dentro do intervalo $\Delta \mathrm{T}_{\mathrm{x}}$ com o intuito de se obter peças com estruturas especiais e de alta resistência mecânica. $\mathrm{O}$ processo de consolidação por extrusão a quente apresenta um grande potencial para a consolidação de fitas ou de pós-provenientes de compósitos de estrutura parte amorfas e parte cristalina, desde que se consiga manter um rigoroso controle dos parâmetros de processo, tais como a temperatura e a taxa de deformação.

\section{Materiais e Métodos}

Os de pós dos elementos alumínio, nióbio e boro, conforme a sua granulometria (peneira de $100 \mathrm{mesh}$ ), tendo uma pureza de 99,9\% cada um dos elementos, provenientes da Aldrich Chemical, foram pesados nas proporções adequadas à composição $\left(\mathrm{Al}_{54} \mathrm{Nb}_{45} \mathrm{~B}_{5}\right)$. Os pós foram pesados em uma balança analítica, com resolução de 10${ }^{4} \mathrm{~g}$, para obter a respectiva composição nominal da liga de $\mathrm{Al}_{54} \mathrm{Nb}_{45} \mathrm{~B}_{5}$. O processo de moagem de alta energia foi realizado a seco em um moinho de bolas planetário Fritsch P-5 sob atmosfera de argônio, usando esferas (19 $\mathrm{mm}$ de diâmetro) e vaso $(225 \mathrm{~mL})$ de aço inoxidável, velocidade de $300 \mathrm{rpm}$ e uma relação de massas esferas/pós de 10:1. Este procedimento foi realizado por $10 \mathrm{~h}$. Diante da ocorrência de mecanismos de soldagem a frio excessiva durante o processo de moagem de alta energia de partículas dúcteis sobre as superfícies do vaso e das esferas, optou-se pela continuação da moagem em meio líquido (álcool isopropílico) por mais $1 \mathrm{~h}$, para a recuperação dos pós previamente soldados. Para a identificação das fases da liga a amorfa $\left(\mathrm{Al}_{54} \mathrm{Nb}_{45} \mathrm{~B}_{5}\right)$, foi usado um difratômetro Shimadzu XRD 6000, utilizando radiação de $\mathrm{CuK \alpha}$ com comprimento de onda $(\lambda=1,5406 \AA)$. As medidas foram tomadas para uma ampla gama de ângulos de difração $(2 \theta)$ que variam de $20^{\circ}$ a $120^{\circ}$ com passo angular de $0,05^{\circ}$ e com tempo de contagem por ponto igual a $2,4 \mathrm{~s}$. A microscopia de varredura eletrônica (MEV) foi obtida utilizando um microscópio FEI-COMPANY modelo QUANTA 500 com tensão de 30 kV. Para melhorar a condutividade das amostras, estas foram recobertas com fina camada de ouro. A análise química foi realizada através por Espectroscopia de Energia Dispersiva (EDS).
A evolução microestrutural do pó durante a moagem foi investigada via Difração de Raios-X e Microscopia Eletrônica de Varredura (MEV). A difração de raios $\mathrm{x}$ foi utilizada para identificar as fases presentes dos pós-obtidos por moinho de alta energia com e sem tratamento térmico.

\section{Resultados e Discussão}

\section{Morfologia da Partícula}

$\mathrm{Na}$ Figura 3 a morfologia das partículas foi investiga pela microscopia eletrônica de varredura (MEV), em todos os tempos de moagem, e foi possível verificar que apesar da irregularidade e a variação do tamanho das partículas do pó, o resultado final não foi influenciado.

Figura 1. Morfologia das partículas do pó da liga de $\mathrm{Al}_{54} \mathrm{Nb}_{45} \mathrm{~B}_{5}$ em a) $5 \mathrm{~h} \mathrm{e} \mathrm{b)} 15 \mathrm{~h}$.

a)

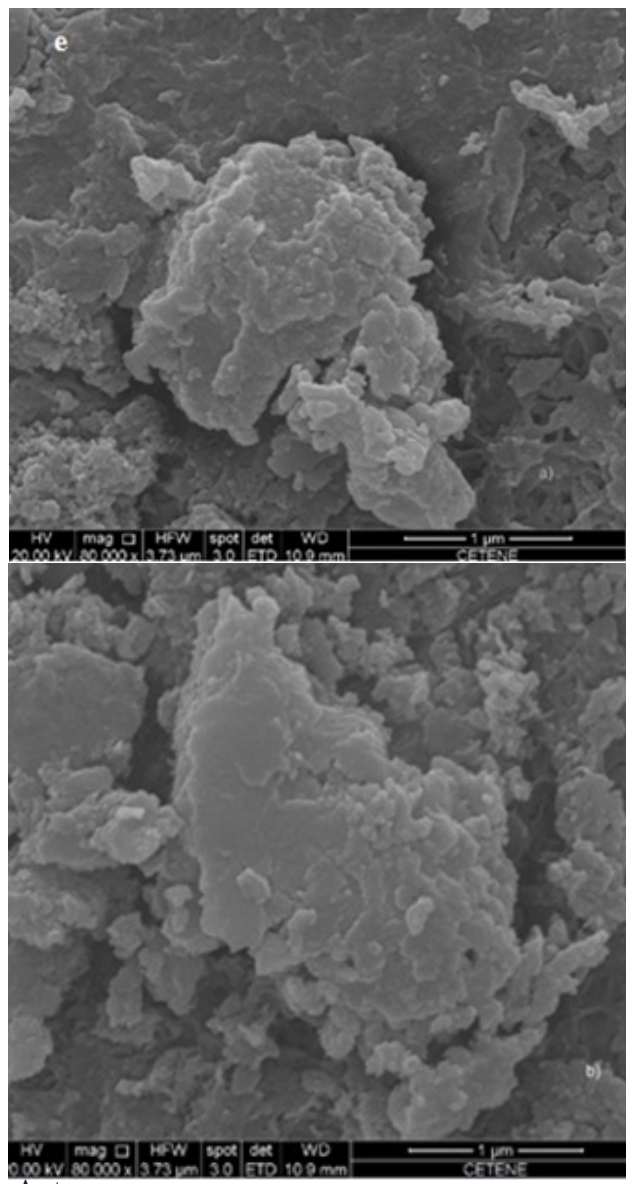

Fonte: Autor 
Essa variação no tamanho das partículas é inerente à moagem que decorre entre o processo de deformação seguido de soldagem e quebra das partículas. É observado um empilhamento, em camadas, de partículas que foram deformadas e soldadas uma sobre outra no processo da Moagem de Alta Energia para melhor refinamento do grão e da sua microestrutura. É observado que aumentando o tempo de moagem ocorre um refinamento das partículas do pó, refinamento esse, que é evidenciado no fim da moagem, i. e., na amostra com $5 \mathrm{~h}$ e $15 \mathrm{~h}$ conforme a moagem, no MEV da Figura 1,com um aumento de $10^{4}$ vezes observase um aglomerado de partículas irregulares e muito finas em relação ao inicio da moagem. Apesar de um refino tão acentuado o processo ainda continua com o empilhamento de partículas deformadas e soldadas de forma sobreposta.

\section{Evolução Microestrutural}

O difratograma de raios x na Figura 2 apresenta as fases em soluções sólidas $\mathrm{S}$ e com complexos metálicos de alumínio (Al) (nas posições $2 \theta$ de $43^{\circ}$ e $67^{\circ}$ de estrutura tetragonal), $\varepsilon-\mathrm{NbB}_{2}$ (nas posições $2 \theta$ de $32^{\circ}$ e $42^{\circ}$ de estrutura hexagonal) do binário $\varphi-\mathrm{Nb}_{5} \mathrm{~B}_{2}$. E do binário $\omega-\mathrm{Al}_{2} \mathrm{Nb}_{3}$ a fase existente é o $\delta$-AlB ${ }_{2}$ de estrutura hexagonal na posição $2 \theta$ de $45^{\circ}$ sobreposto ao pico do $\mathrm{Al}$ (JUNG et al.,2009). As fases presentes são na sua maioria intermetálicos dos binários $\mathrm{Nb}-\mathrm{B}$ e Al-B, e a solução sólida do Alumínio no Nióbio.

Com o tratamento térmico as fases da solução sólida e do alumínio puro tendem a se transformar em fases intermetálicas estáveis, com a evolução do tempo de moagem. O processo de difusão com que os átomos de Boro, amorfiza a estrutura para elétrons que migre com maior facilidade para as redes cristalinas do Nióbio e do alumínio deformando a rede e formando as fases intermetálicas dos binários $\mathrm{AlB}$ e $\mathrm{NbB}$ ,pois o aumento do tempo de moagem refina a microestrutura da liga $\mathrm{Al}_{54} \mathrm{Nb}_{45} \mathrm{~B}_{5}$.

Figura 2. Difratograma de Raios $X$ da evolução da liga amorfa $\mathrm{Al}_{54} \mathrm{Nb}$

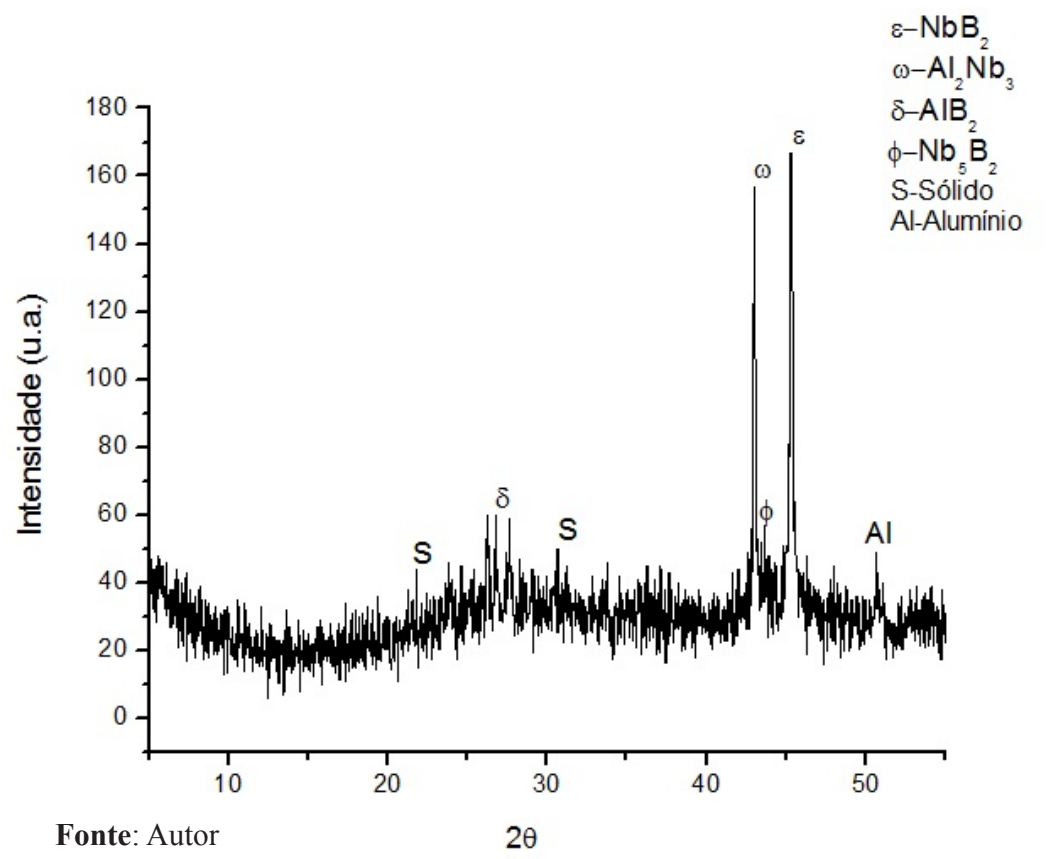

Essa solução sólida do Alumínio no Nióbio é comprovada pelo aumento do parâmetro de rede do nióbio. Na Figura 3 pode-se observar a variação do parâmetro de rede do $\mathrm{Nb}$ com o tempo de moagem. 
Figura 3. Evolução do parâmetro de rede do $\mathrm{Al}$ e $\mathrm{Nb}$ da liga $\mathrm{Al}_{54} \mathrm{Nb}_{45} \mathrm{~B}_{5}$

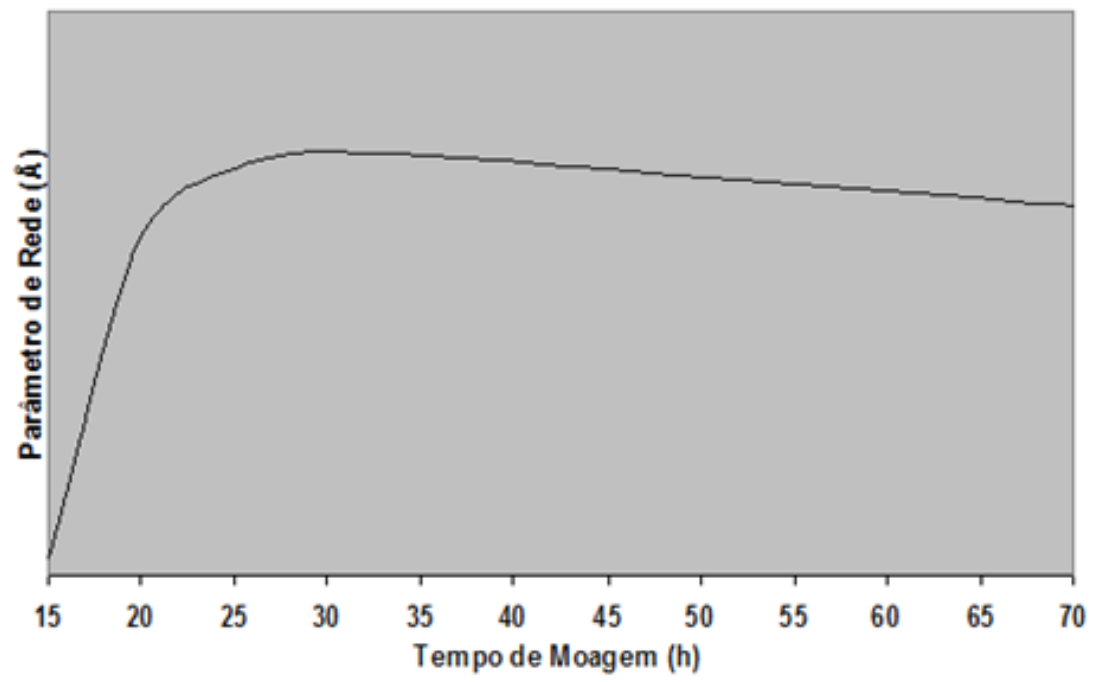

Fonte: Autor

Os átomos de alumínio são introduzidos na rede cristalinadoNióbioalongandoseuparâmetroderede até um determinado tempo, que ainda permanece cúbica, e em seguida tem uma leve diminuída pela continuação do processo da moagem, conforme a Figura 3. A formação de uma solução sólida indica que a liga esta atingindo um maior grau de homogeneização. O EDS da Figura 4 mostra uma região amostra rica em Al e $\mathrm{Nb}$ com presença do $\mathrm{B}$ amorfizando a liga $\mathrm{Al}_{54} \mathrm{Nb}_{45} \mathrm{~B}_{5}$ de maiores concentração de Alumínio, e Nióbio se complexando com Oxigênio e B onde existem regiões ricas em Nióbio com presença de intermetálicos. A presença da fase amorfa na amostra devido à pequena porcentagem de fase amorfa presente, sendo que a intensidade dos picos de $\mathrm{Al}$-fcc e $\mathrm{Nb}$-fcc são muito superiores quando comparados aos correspondentes à fase amorfa, isto por que a composição da fase amorfa é induzida pelo o B que amorfiza o sistema e forma intermetálicos junto com o $\mathrm{Nb}$, alterando a difusão de $\mathrm{Al}$ da fase amorfa para a fase cristalina na $\mathrm{Al}_{54} \mathrm{Nb}_{45} \mathrm{~B}_{5}$ pelas maiores concentrações vista no EDS.

Figura 4. EDS da amostra rica em $\mathrm{Al}$ e $\mathrm{Nb}$ com presença do $\mathrm{B}$ amorfizando a liga $\mathrm{Al}_{54} \mathrm{Nb}_{45} \mathrm{~B}_{5}$

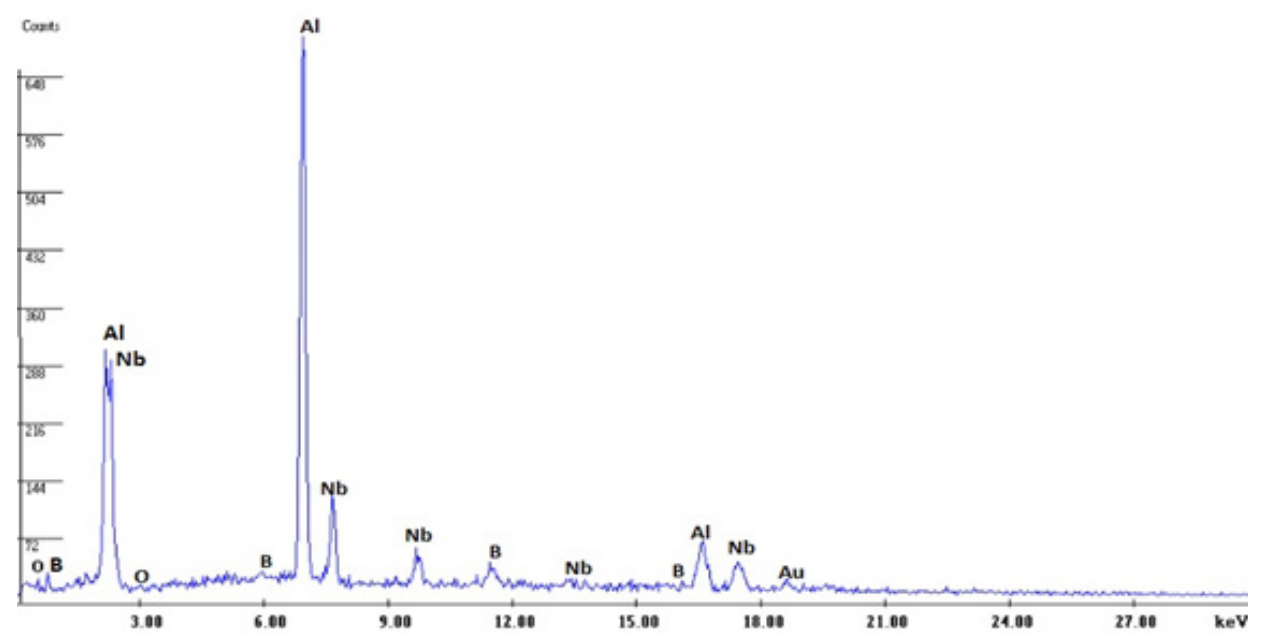

Fonte: Autor 


\section{Conclusão}

O processo por moinho de alta energia com os parâmetros utilizados mostrou-se eficiente na obtenção da liga Al-Nb-B coma amorfização da mesma como foi observado no difratograma de raios- $\mathrm{x}$ com fases intermetálicas.

A partir de $5 \mathrm{~h}$ e $15 \mathrm{~h}$ de moagem há formação de uma fase desconhecida rica em Nióbio que foi caracterizada como uma solução sólida do Alumínio no Nióbio, se complexando com o Boro constatando a solubilidade do Nióbio na liga. Essa fase ainda permanece com estrutura cúbica original do Nióbio, porém com seu parâmetro de rede alongado pela introdução do Alumínio na rede.

A moagem proporcionou, além da formação de liga, uma morfologia com partículas refinadas com formatos irregulares. Esse refino é observado não apenas em proporções macro, mas também microestrutural, indicando uma distribuição homogênea de partículas de segunda fase que permitiram um refinamento nos grãos de alumínio. $\mathrm{O}$ processamento de refinamento e parâmetros utilizados é suficientemente bom para fabricar a liga $\mathrm{Al}-\mathrm{Nb}-\mathrm{B}$, porque o Nióbio é completamente dissolvido (solução de fases termodinâmicas), juntamento com o Boro que amorfiza as regiões junto com o Alumínio, criando regiões de fases intermetálicas e amorfas. No entanto, a optimização da adição de pó de $\mathrm{Nb}$ para prevenir a sua oxidação, melhor B, tendo maior resistência à corrosão; Este trabalho também demonstra que a adição destas ligas mestre Al-Nb-B para alumínio e suas ligas introduz potentes inoculantes $\mathrm{Nb}-\mathrm{B}$, que promovem o refinamento da estrutura de grãos via nucleação heterogênea. $\mathrm{Nb}-\mathrm{B}$ é eficaz na inoculação diferente de Al-Si fundido em ligas solidificou-se sobvárias temperaturas e em condições de resfriamento.

\section{Agradecimentos}

Os autores agradecem ao PRH 28 / MCT / ANP pelo o apoio financeiro deste trabalho e ao Laboratório de Magnetismo e Materiais Magnéticos-MMM do
Departamento de Física do Centro de Ciências Exatas e da Natureza da UFPE, e ao CETENE.

\section{Referências}

AL-ZAIN, Y.; KIM, Y. H. MIYAZAKI, S. Effect of $\mathrm{B}$ addition on the microstructure and superelastic properties of aTi-26Nb alloy. Materials Science \& Engineering A, n. 644, p. 85-89, 2015.

BOLZONI, L.; NOWAK, M.; BABU, N. H. Assessment of the influence of Al-2Nb-2B master alloy on the grain refinement and properties of LM 6 (A413) alloy. Materials Science \& Engineering A, n. 628, p. $230-237,2015$.

BAREKAR, N. Effect of melt-spinning process parameters on the microstructure of Al-Ni-La alloys, Master thesis, Indian Institute of Technology, 2006.

CAI, B.; ZHENG, Z. Q.; HE, D. Q.; LI, S. C.; LI, H. P. Friction stir weld of 2060 Al-Cu-Li alloy: Microstructure and mechanical properties. Journal of Alloys and Compounds, n. 649, p. 19-27, 2015.

HONO, K.; ZHANG,Y.; TSAI, A. P.; INOUE, A.; SAKURAI, T. Solute partitioning in partially crystallized Al-Ni-Ce(-Cu) metallic glass. Scripta Metallurgica et Materialia, n. 32, p. 191-196, 1995.

INOUE, A. Amorphous, nanoquasicrystalline and nanocrystalline alloys in Al-based systems. Progress in Materials Science, n. 43, p. 365-520, 1998.

JIA， M. T.; ZHANG， D. L.; GABBITAS，B.; LIANG, J. M.; KONG, C. A novel Ti-6Al-4V alloy microstructure with very high strength and good ductility. Scripta Materialia, n. 107, p.10-13, 2015.

JUNG, H.; MANGELINCK-NOËL， N.; BERGMAN, C.; BILLIA, B. Determination of the average nucleation undercooling of primary Alphase on refining particles from $\mathrm{Al}-5.0 \mathrm{wt} \% \mathrm{Ti}-1.0$ $\mathrm{wt} \% \mathrm{~B}$ in Al-based alloys using DSC. Journal of Alloys and Compounds, n. 477, p. 622-627, 2009.

LI,Y. L.; FENG,H. K.; CAO, F. R.; CHENA,Y. B.; GONG, L.Y. Effect of high density ultrasonic on the microstructure and refining property of $\mathrm{Al}-5 \mathrm{Ti}-$ $0.25 \mathrm{C}$ grain refiner alloy. Materials Science and Engineering A, n. 487, p. 518-523, 2008. 
MCCARTNEY, D. G. Grain refining of aluminium and its alloys using inoculants. Int. Mater. Ver., n. 34, p. 247-60, 1980.

NASCIMENTO, L. Estudo das Ligas Amorfas na Reação de Oxidação com Aplicabilidade na síntese do Metanol e Produção de Olefinas. 2013. Projeto de Tese de Doutorado (Doutorado em Engenharia Química) Universidade Federal de Pernambuco, Recife-PE.

NOWAK, M.; BOLZONI, L.; BABU, N. H. Grain refinement of Al-Si alloys by $\mathrm{Nb}-\mathrm{B}$ inoculation. Part I: Concept development and effect on binary alloys. Materials and Design, n. 66, p. 366-375, 2015.

NOWAK, M.; YEOH, W. K.; BOLZONI, L.; BABU, N. H. Development of Al-Nb-B master alloys using $\mathrm{Nb}$ and $\mathrm{KBF}_{4}$ Powders. Materials and Design, 75, p. 40-46, 2015a.

SAHOO, K. L.; WOLLGARTEN, M.; KIM, K. B.; BANHART, J. Crystallization behavior and microhardness evolution in $\mathrm{Al}_{92-\mathrm{x}} \mathrm{Ni}_{8} \mathrm{La}_{\mathrm{x}}$ amorphous alloys. Journal of Materials Research, n. 20, p. 2927-2933, 2005.

SIGWORTH, G. K. The grain refining of aluminum and phase relationships in the Al-Ti-B system. Metall. Trans. A, n. 15, p. 277-82, 1984. 